\title{
Vor- und Nachteile der Kastration von Kätzinnen und Katern
}

Reichler, I M

\begin{abstract}
Die Entfernung der Gonaden bewirkt durch den Wegfall von Keimzellen und den Sexualsteroiden eine irreversible Verhinderung der Reproduktion. Folge der Gonadektomie sind aber auch metabolische Veränderungen, die einen Einfluss auf die Inzidenz von Krankheiten sowie auf das äussere Erscheinungsbild und das Verhalten haben. Diese Vorund Nachteile der Kastration werden in Abhängigkeit vom Zeitpunkt der Kastration auf die verschiedenen Organsysteme bei Kätzinnen und Katern dargestellt. Aus Gründen der Krankheitsprophylaxe als auch der Haltungserleichterung und der sogenannten „pet overpopulation" ist die Kastration von Katzen, die nicht zur Zucht vorgesehen sind, bereits vor der Pubertät zu empfehlen. Es sollte dabei auf das Risiko für Übergewicht und damit verbundenen Erkrankungen hingewiesen sowie eine Diätberatung durchgeführt werden.
\end{abstract}

DOI: https://doi.org/10.1024/0036-7281/a000064

Other titles: Pros and Cons of gonadectomy in female and male cats

Posted at the Zurich Open Repository and Archive, University of Zurich

ZORA URL: https://doi.org/10.5167/uzh-43918

Journal Article

Accepted Version

Originally published at:

Reichler, I M (2010). Vor- und Nachteile der Kastration von Kätzinnen und Katern. Schweizer Archiv für Tierheilkunde, 152(6):273-278.

DOI: https://doi.org/10.1024/0036-7281/a000064 
Vor- und Nachteile der Kastration von Kätzinnen und Katern

I.M. Reichler, Klinik für Fortpflanzungsmedizin der Universität Zürich

Die Entfernung der Gonaden bewirkt durch den Wegfall von Keimzellen und den Sexualsteroiden eine irreversible Verhinderung der Reproduktion. Folge der Gonadektomie sind aber auch metabolische Veränderungen, die einen Einfluss auf die Inzidenz von Krankheiten sowie auf das äussere Erscheinungsbild und das Verhalten haben. Diese Vorund Nachteile der Kastration werden in Abhängigkeit vom Zeitpunkt der Kastration auf die verschiedenen Organsysteme bei Kätzinnen und Katern dargestellt. Aus Gründen der Krankheitsprophylaxe als auch der Haltungserleichterung und der sogenannten ,pet overpopulation“ ist die Kastration von Katzen, die nicht zur Zucht vorgesehen sind, bereits vor der Pubertät zu empfehlen. Es sollte dabei auf das Risiko für Übergewicht und damit verbundenen Erkrankungen hingewiesen sowie eine Diätberatung durchgeführt werden.

Schlüsselwörter: Kastration, Kastrationszeitpunkt, Kater, Kätzin

Pros and Cons of gonadectomy in female and male cats

Gonadectomy irreversibly prevents reproduction by removing germ cells as well as gonadal hormones. Moreover, consequences of gonadectomy include metabolic alterations influencing incidence of diseases, phenotype and behavior. The desirable and undesirable effects of castration on different organ systems and their relation to timing of gonadectomy are discussed. For reasons of disease prevention as well as ease of husbandry and control of pet overpopulation prepuberal castration of non-breeding cats is recommended. At the time of gonadectomy the risk for developing obesity and associated diseases as well as dietary measures should be discussed with the owner.

Keywords: gonadectomy, timing of gonadectomy, tom cat, queen 


\section{Einleitung}

Die chirurgische Entfernung der Gonaden bewirkt durch den Wegfall der germinativen und endokrinen Funktion eine irreversible Verhinderung der Reproduktion. Durch das Fehlen der Gonadenhormone ist auch das Sexualverhalten stark reduziert. Aufzeichnungen aus dem 18. Jahrhundert zeigen, dass die Beeinflussung des Sexualverhaltens bereits damals Grund für die Kastration von Katern war. So antwortet eine Italienerin ihrer französischen Freundin auf die Frage, was sie denn mit einer kastrierten Katze anfange, „Lo tengo per non guastarmi la vita (Ich halte ihn so, um mein Leben nicht zu stören, Barbier, 1995). Auch in der heutigen Zeit dürfte beim Kater die Verhinderung geschlechtsspezifischen Verhaltens wie das Harnmarkieren und der typische Katergeruch an erster Stelle stehen. Bei Kätzinnen, die nur im Haus gehalten werden, ist vermutlich die Ausschaltung der Raunze der häufigste Kastrationsgrund, während bei Freigängerinnen dies die Reproduktionskontrolle ist.

Der Wegfall der Gonadenhormone (Östrogen, Testosteron, Progesteron) und die vermehrte Ausschüttung von FSH und LH beeinflussen zahlreiche Stoffwechselprozesse. Als Folge der Kastration kann bei Kater und Kätzin auch ein Anstieg von Prolaktin, Leptin und Insulin-like growth factor 1 beobachtet werden. Die damit verbundenen metabolischen Veränderungen beeinflussen die Häufigkeit von Krankheiten, wirken aber auch auf das äussere Erscheinungsbild und das Verhalten. Im Folgenden werden die Auswirkungen der Gonadektomie in Abhängigkeit vom Zeitpunkt der Kastration auf verschiedene Organsysteme dargestellt.

\section{Auswirkungen der Kastration auf den Reproduktionstrakt bei der Kätzin}

Uteruserkrankungen und Ovarialzysten sind ein häufiges Problem bei intakten Kätzinnen, wohingegen Tumoren und Entzündungen der Gonaden sehr selten auftreten (Potter et al., 1991). Die Kastration wirkt präventiv auf Ovar- und Uteruserkrankungen, da sowohl Hydround Mucometra als auch der glandulärzystische Hyperplasie-/Pyometrakomplex über gonadale Hormone vermittelt werden. Besteht bereits eine Metropathie, so ist die Ovariohysterektomie die empfohlene Behandlung. Eine elektive Ovariohysterektomie wird in unserer Klinik nur bei älteren Tieren oder bei Tieren, die mit Gestagenen behandelt wurden, durchgeführt, da in diesen Fällen möglicherweise bereits eine Uteruserkrankung vorliegt. Ansonsten wird die Ovariektomie bevorzugt, da dieser Eingriff weniger invasiv ist und Vorteile der Entfernung eines gesunden Uterus bisher nicht bekannt sind. 
Die Entwicklung und Größe des äusseren Genitales ist von den Geschlechtshormonen abhängig. Werden Kätzinnen vor Erreichen der Pubertät kastriert, bleibt die Vulva lebenslang klein und infantil, diese Unreife hat jedoch keine klinische Konsequenz (Root et al., 1996).

\section{Mammatumoren}

Bei der Kätzin zählen Mammatumoren mit einer Inzidenz von 17\% zu den dritthäufigsten Tumoren (Schmidt und Langham, 1967; Dorn et al., 1968; Patnaik et al., 1975; Hayes et al., 1981). Der überwiegende Anteil (> 85\%) der felinen Mammatumoren ist maligne (Schmidt und Langham, 1967; Hayes et al., 1981; Bostock, 1986). Wie bei der Hündin besteht auch bei der Katze ein präventiver Effekt der Kastration auf die Entwicklung maligner Mammatumoren: Werden Kätzinnen kastriert bevor sie 6 Monate alt sind, beträgt die Risikoreduktion 91\%, bei Kastration zwischen dem Alter von einem halben und einem Jahr oder zwischen einem und zwei Jahren beträgt die Risikoreduktion nur noch 89\% bzw. 11\%. Eine Kastration nach dem 2. Lebensjahr hat keinen präventiven Effekt auf die Entstehung maligner feliner Mammatumoren (Overley et al., 2005).

\section{Auswirkungen der Kastration auf den Reproduktionstrakt beim Kater}

Tumoren und Entzündungen der Gonaden sind beim Kater sehr selten, ebenfalls Erkrankungen der Nebenhoden, Prostata und Bulbourethraldrüsen. Die Kastration verhindert Erkrankungen der Gonaden und wirkt prävenitv auf entzündliche Erkrankungen der akzessorischen Geschlechtsdrüsen. Im Unterschied zum Hund ist bisher kein deutlicher Zusammenhang zwischen dem Kastrationsstatus und dem Auftreten von Prostatatumoren bekannt, da diese Erkrankungen extrem selten und die Mehrheit aller untersuchten Kater kastriert sind. Bisher wurden Prostatatumoren bei insgesamt 8 Katern beschrieben, 7 der Kater waren kastriert (Hawe, 1983; Carpenter et al., 1987; Hubbard et al., 1990; Caney et al., 1998; LeRoy und Lech, 2004).

Die Entwicklung und Größe des äusseren Genitales ist von Testosteron abhängig. Bei Katern, die im Welpenalter oder vor der Pubertät kastriert werden, bleiben Präputium und Penis klein und unreif, häufig kann der Penis nicht ausgeschachtet werden (Root et al., 1996).

DieseUnreife ist jedoch klinisch nicht von Bedeutung. Testosteron ist nicht nur für die normale Entwicklung von Penis und Präputium, sondern auch für die Ausbildung der Penishäkchen notwendig. Das Vorhandensein von Penishäkchen eignet sich daher als Nachweis der Testosteronsekretion und damit von Hodengewebe. Anhand dieses sekundären 
Geschlechtsmerkmals kann ein beidseits kryptorchider Kater von einem kastrierten Kater unterschieden werden.

\section{Auswirkungen der Kastration auf andere Organsysteme}

\section{Ableitende Harnwege}

Die Auswirkung der Kastration auf den felinen Harntrakt wird kontrovers diskutiert. In einer der Studien (Lekcharoensuk et al., 2001) wurden Gonadektomie und Obesitas als Risikofaktoren für die Entstehung von unteren Harnwegserkrankungen ausgewiesen. Hingegen wurde in einer anderen prospektiv durchgeführten Studie (Root et al., 1996) bei Katzen im Alter von 22 Monaten kein Unterschied in der urethralen Funktion festgestellt, unabhängig davon ob sie im Welpenalter oder später kastriert wurden. Es zeigte sich jedoch, dass die präpelvische Urethra von bereits im Welpenalter kastrierten Kätzinnen einen kleineren Durchmesser aufwies als die intakter Kätzinnen (Root et al., 1996). Beim Kater wurde diesbezüglich kein Unterschied festgestellt (Herron, 1970; Herron 1972; Root 1995). In einer Untersuchung (Howe et al., 2000), in der die Langzeitauswirkung der Kastration im Welpenalter mit der einer später erfolgten Kastration verglichen wurde, konnte bezüglich Häufigkeit der unteren Harnwegserkrankungen kein Unterschied gefunden werden.

Fettstoffwechsel und Pankreas

Obesitas wird durch Faktoren wie Fütterung, Rasse und/oder Aktivität beeinflusst und tritt sowohl bei kastrierten als auch intakten Katzen auf. Die Kastration ist aber bei Katzen eindeutig ein Risikofaktor für Obesitas, denn für kastrierte Katzen ist die Wahrscheinlichkeit übergewichtig zu werden 3.4 mal grösser als für intakte Katzen (Kanchuk et al., 2002; Nguyen et al., 2004). Die Zunahme des Körpergewichts nach Kastration scheint die Folge einer gesteigerten Futteraufnahme zu sein. Die Gewichtszunahme geht mit einer Veränderung der Körperzusammensetzung, insbesondere einer Steigerung des Körperfettanteils, einher. Als Ursache für die Gewichtszunahme nach Kastration werden erhöhte Werte von IGF-1 und Prolaktin diskutiert, da beide Hormone and der Bildung und Aufrechterhaltung des Fettgewebes beteiligt sind (Martin und Siliart, 2005). Obesitas ist jedoch keine zwingende Folge der Gonadektomie. Einfache Massnahmen wie Futterumstellung und Aktivitätssteigerung können die Entstehung von Obesitas verhindern. Bei Katzen reicht aber eine Reduktion des Fettgehaltes im Futter bei ad libidum Fütterung als Obesitasprophylaxe nicht aus, die Futtermenge muss ebenfalls reduziert werden (Nguyen et al., 2004). Mit der 
Obesitasprävention werden auch das Risiko für andere Erkrankungen wie Diabetes mellitus, Lahmheiten und nicht allergische Hauterkrankungen gesenkt und somit die Lebenserwartung gesteigert (Scarlett und Donoghue, 1998).

Bei Katzen sind Obesistas aber auch der Kastrationsstatus, Alter und Geschlecht Risikofaktoren für Diabetes mellitus (Panciera et al., 1990). Kastrierte Kater sind nicht nur häufiger übergewichtig, sondern erkranken auch 2-9 mal häufiger an Diabetes mellitus als intakte Katzen (Panciera et al., 1990; McCann et al., 2007; Prahl et al., 2007), vermutlich aufgrund einer reduzierten Insulinsensitivität nach der Kastration (Hoenig und Ferguson, 2002; Kanchuk et al., 2002). Bei fetten Tieren ist auch die Leptinkonzentration im Blut erhöht, die mit dem Grad der Insulinresistenz korreliert und vermutlich ursächlich an der Entstehung von Diabetes mellitus bei kastrierten Katern beteiligt ist (Martin et al., 2001).

\section{Bewegungsapparat}

Bereits seit dem Altertum ist der eunuchoide Hochwuchs als Folge der präpubertären Kastration bekannt. Präpubertär ist das Längenwachstum der Gliedmaßen stärker als das der axialen Knochen. Der Anstieg der Geschlechtssteroide in der Pubertät führt zum Schliessen der Wachstumsfugen und beendet damit das Längenwachstum. Durch eine präpubertäre Kastration wird der Fugenschluss verzögert und als Folge der verlängerten Wachstumsphase entsteht ein dysproportionierter Hochwuchs (Houlton und McGlennon, 1992; Root et al., 1997). Werden Kater mit 7 Wochen oder 7 Monaten kastriert, ist ihr Radius, wenn sie ausgewachsen sind, $13 \%$ länger als der von intakten Katern (Root et al., 1997). Bei Katzen wurde postuliert, dass Femurkopffugenfrakturen im Zusammenhang mit dem verzögerten Fugenschluss nach Frühkastration stehen (McNicholas et al., 2002). Tritt eine Femurkopffugenfraktur ohne bekanntes Trauma auf, ist der betroffene Patient meist ein übergewichtiger kastrierter Kater (Craig, 2001; McNicholas et al., 2002; Fischer et al., 2004), was die Bedeutung der Obesitasprävention bei kastrierten Katzen deutlich unterstreicht. In grossen Populationsstudien (Spain et al., 2004) wurde jedoch kein Zusammenhang zwischen Kastrationszeitpunkt und Frakturinzidenz festgestellt.

Infektionserkrankungen und Erkrankungen des Immunsystems

Bei Katzen werden das feline Immunschwächevirus (FIV) vor allem durch Bisse beim Deckakt und bei Revierkämpfen und das feline Leukämievirus (FeLV) durch engen Kontakt, zum Beispiel durch gemeinsame Nutzung von Fressplätzen übertragen. Die Kastration 
mindert einerseits das Deckverhalten, andererseits aber auch das Territorialverhalten (Kalz, 2001), so dass Fressplätze vermehrt gemeinsam genutzt werden. Ob durch die Kastration das Infektionsrisiko für FIV oder FeLV beeinflusst wird, ist aber nicht beschrieben. Der Zeitpunkt der Kastration scheint die Inzidenz dieser Erkrankungen nicht zu beeinflussen (Howe et al., 2000).

Feline infektiöse Peritonitis (FIP) tritt bei intakten Katzen, vor allem bei intakten Katern häufiger auf als bei kastrierten Tieren (Rohrbach et al., 2001; Pesteanu-Somogyi et al., 2006). Ungeklärt ist jedoch, ob die Haltungsform sowohl die Inzidenz von FIP als auch den Kastrationsstatus beeinflussen kann. Intakte Tiere stammen in der Regel aus Beständen in denen mehrere Katzen zusammengehalten werden und hier ist die Seroprävalenz feliner Coronaviren höher als in Haushalten mit nur einer Katze (Bell et al., 2006a; Bell et al., 2006b). Der Zeitpunkt der Kastration soll keinen Einfluss auf die Erkrankungshäufigkeit haben (Spain et al., 2004).

Gingivitis und Asthma sollen bei älteren Katzen, die bereits im Welpenalter kastriert wurden, seltener auftreten als bei Tieren, die im Alter zwischen 5.5 Monaten und einem Jahr kastriert wurden (Spain et al., 2004).

\section{Lebenserwartung}

Kastrierte Kätzinnen leben im Mittel ein Jahr, kastrierte Kater sogar 4 Jahre länger als ihre intakten Geschlechtsgenossen (Kraft, 1998). Inwieweit die längere Lebenserwartung Folge einer krankheitspräventiven Wirkung der Kastration ist bleibt ungeklärt. Kätzinnen und Kater, die als Familienmitglieder gehalten werden und medizinisch auch als soche versorgt werden, sind in der Regel kastriert. Intakten Freiläufern wird dagegen meist weniger Aufmerksamkeit und medizinische Fürsorge gewidmet.

Verhalten

Die Gonadektomie von Katzen, unabhängig davon ob sie bereits im Welpenalter oder um die Pubertät herum erfolgte, mindert das Aggressionsverhalten gegenüber Artgenossen und steigert die Anhänglichkeit gegenüber Menschen. Spielfreude, Erregbarkeit oder Häufigkeit der Vokalisation werden dagegen nicht beeinflusst (Stubbs et al., 1996). Unterschiedlich diskutiert wird der Einfluss des Kastrationzeitpunkts. Während in einer grossen Populationsstudie (Spain et al., 2004) die später kastrierten Katzen im Vergleich zu im 
Welpenalter kastrierten Tieren häufiger hyperaktiv waren, stellten andere Studien (Stubbs et al., 1996; Wright und Amoss, 2004) diesbezüglich keinen Unterschied fest.

Urinmarkieren wird durch die Kastration gemindert. Unabhängig davon, ob Kätzinnen oder Kater zum Zeitpunkt der Kastration bereits Markierverhalten zeigten, markieren nach der Kastration rund 5\% der Kätzinnen und 10\% der Kater, wenn sie zum Zeitpunkt der Kastration ein halbes Jahr oder älter waren (Hart und Cooper, 1984; Wright und Amoss, 2004). Kater, die bereits im Welpenalter kastriert werden, markieren dagegen seltener, sie zeigen allgemein weniger geschlechtsspezifisches Verhalten und haben daher auch seltener Abszesse (Spain et al., 2004). Der präventive Effekt der Kastration im Welpenalter auf die Abszessinzidenz schwindet jedoch mit zunehmendem Lebensalter (Spain et al., 2004).

\section{Kastration zur Kontrolle der Reproduktion}

Wegen der Irreversibilität der chirurgischen Kastration werden Kastrationsaktionen weltweit zur Bekämpfung der Katzenüberbevölkerung durchgeführt. Zahlen aus den USA zeigen jedoch, dass trotz hoher Kastrations- und Tötungsraten die Katzenpopulation um 2.9\% pro Jahr weiter zunimmt (New, 2006). Ähnliche Angaben liegen auch für Europa vor: In der Stadt Berlin beispielsweise werden seit über 10 Jahren jährlich zwischen 5000 und 10000 verwilderte Katzen eingefangen, kastriert und am Fangort wieder ausgesetzt, dennoch wurde keine nachhaltige Reduktion der Tierzahl festgestellt (Kalz, 2001). Selbst wenn zwei Drittel der weiblichen Katzen kastriert sind, nimmt die Populationsgrösse zu und wenn alle weiblichen Katzen in einem Gebiet kastriert werden, erfolgt eine Zuwanderung fremder Katzen. Die Kastration oder der Tod von Deckkatern wirken sich auf die Gesamtpopulation nicht aus, da fremde Kater zuwandern und für den Ausgleich sorgen (Kalz, 2001).

Zur Kontrolle der Katzenüberbevölkerung wird nach einer einmalig und einfach anzuwendenden Massnahme gesucht, die wie die Kastration zur permanenten Kontrazeption führt, jedoch weniger aufwändig und deutlich billiger ist (Peters, 2008). Bis bessere Methoden zur Populationskontrolle zur Verfügung stehen, empfiehlt sich weiterhin die chirurgische Kastration von Kätzinnen, um zu hohe Populationsdichten, Tierleid und die Ausbreitung von Zoonosen zu vermeiden. Auch die Kastration von Katern ist weiterhin empfohlen, da kastrierte Tiere einen durchschnittlich besseren Gesundheitsstatus und ein geringeres Mortalitätsrisiko haben als unkastrierte (Kalz, 2001). 
Eine Kastration im Welpenalter führt weder zu nachteiligem Verhalten noch zu einer höheren Krankheitsrate und kann deshalb beim Kater und bei der Kätzin empfohlen werden.

\section{Literatur:}

Barbier P.: Farinelli. Der Kastrat der Könige. Econ, Berlin, 1995.

Bell E. T., Malik R., Norris J. M.: The relationship between the feline coronavirus antibody titre and the age, breed, gender and health status of Australian cats. Aust. Vet. J. 2006a, 84:27.

Bell E. T., Toribio J. A., White J. D., Malik R., Norris J. M.: Seroprevalence study of feline coronavirus in owned and feral cats in Sydney, Australia. Aust. Vet. J. 2006b, 84:74-81.

Bostock D. E.: Canine and feline mammary neoplasms. Br .Vet. J. 1986, 142:506-515.

Caney S. M., Holt P. E., Day M. J., Rudorf H., Gruffydd-Jones T. J.: Prostatic carcinoma in two cats. J. Small Anim. Pract. 1998, 39:140-143.

Carpenter J., Andrews L., Holsworth J.: Tumors and tumor-like lesions. In: Diseases of the cat. Ed.. J. Holzworth, WB Saunders, Philadelphia, 1987, 406-411.

Craig L. E.: Physeal Dysplasia with Slipped Capital Femoral Epiphysis in 13 Cats. Vet. Pathol. 2001, 38:92-97.

Dorn C. R., Taylor D. O., Schneider R., Hibbard H. H., Klauber M. R.: Survey of animal neoplasms in Alameda and Contra Costa Counties, California. II. Cancer morbidity in dogs and cats from Alameda County. J. Natl. Cancer Inst. 1968, 40:307-318.

Fischer H. R., Norton J., Kobluk C. N., Reed A. L., Rooks R. L., Borostyankoi F.: Surgical reduction and stabilization for repair of femoral capital physeal fractures in cats: 13 cases. J. Am. Vet. Med. Assoc. 2004, 224:1478-1482.

Hart B. L., Cooper L.: Factors relating to urine spraying and fighting in prepubertally gonadectomized cats. J. Am. Vet. Med. Assoc.1984, 184:1255-1258.

Hawe R. S.: What is your diagnosis? Prostatic adenocarcinoma. J. Am. Vet. Med. Assoc.1983, 182:1257-1258.

Hayes H. M., Jr., Milne K. L., Mandell C. P.: Epidemiological features of feline mammary carcinoma. Vet. Rec. 1981, 108:476-479.

Hoenig M., Ferguson D. C.: Effects of neutering on hormonal concentrations and energy requirements in male and female cats. Am. J. Vet. Res. 2002, 63:634-639.

Houlton J. E., McGlennon N. J.: Castration and physeal closure in the cat. Vet. Rec. 1992, 131:466-467. 
Howe L. M., Slater M. R., Boothe H. W., Hobson H. P., Fossum T. W., Spann A. C., Wilkie W. S.: Long-term outcome of gonadectomy performed at an early age or traditional age in cats. J. Am. Vet. Med. Assoc. 2000, 217:1661-1665.

Hubbard B. S., Vulgamott J. C., Liska W. D.: Prostatic adenocarcinoma in a cat. J. Am. Vet. Med. Assoc.1990, 197:1493-1494.

Kalz B.: Populationsbiologie, Raumnutzung und Verhalten verwildeter Hauskatzen und der Effekt von Maßnahmen zur Reproduktionskontrolle. Dissertation, Humboldt-Universität Berlin, 2001.

Kanchuk M. L., Backus R. C., Calvert C. C., Morris J. G., Rogers Q. R.: Neutering induces changes in food intake, body weight, plasma insulin and leptin concentrations in normal and lipoprotein lipase-deficient male cats. J. Nutr. 2002, 132:1730S-1732S.

Kraft W.: Geriatrics in canine and feline internal medicine. Eur. J. Med. Res. 1998, 3:31-41.

Lekcharoensuk C., Osborne C. A., Lulich J. P.: Epidemiologic study of risk factors for lower urinary tract diseases in cats. J. Am. Vet. Med. Assoc. 2001, 218:1429-1435.

LeRoy B. E., Lech M. E.: Prostatic carcinoma causing urethral obstruction and obstipation in a cat. J. Feline Med. Surg. 2004, 6:397-400.

Martin L., Siliart B., Dumon H., Backus R., Biourge V., Nguyen P.: Leptin, body fat content and energy expenditure in intact and gonadectomized adult cats: a preliminary study. J. Anim. Physiol. Anim. Nutr. (Berl). 2001, 85:195-199.

Martin L. J., Siliart B. S.: Hormonelle Folgen der Kastration bei Katzen. Waltham Focus. 2005, 15:32-35.

McCann T. M., Simpson K. E., Shaw D. J., Butt J. A., Gunn-Moore D. A.: Feline diabetes mellitus in the UK: the prevalence within an insured cat population and a questionnaire-based putative risk factor analysis. J. Feline Med. Surg. 2007, 9:289-299.

McNicholas W. T., Jr., Wilkens B. E., Blevins W. E., Snyder P. W., McCabe G. P., Applewhite A. A., Laverty P. H., Breur G. J.: Spontaneous femoral capital physeal fractures in adult cats: 26 cases (1996-2001). J. Am. Vet. Med. Assoc. 2002, 221:1731-1736.

New J.: The Math, Myth and Management of Pet Population: Births and Deaths of Dogs and Cats in the U.S. Third International Symposium on Non-Surgical Contraceptive Methods for Pet Population Control, Alexandria, Virginia, US 2006.

Nguyen P. G., Dumon H. J., Siliart B. S., Martin L. J., Sergheraert R., Biourge V. C.: Effects of dietary fat and energy on body weight and composition after gonadectomy in cats. Am. J. Vet. Res. 2004, 65:1708-1713. 
Overley B., Shofer F. S., Goldschmidt M. H., Sherer D., Sorenmo K. U.: Association between ovariohysterectomy and feline mammary carcinoma. J. Vet. Intern. Med. 2005, 19:560-563. Panciera D. L., Thomas C. B., Eicker S. W., Atkins C. E.: Epizootiologic patterns of diabetes mellitus in cats: 333 cases (1980-1986). J. Am. Vet. Med. Assoc. 1990, 197:1504-1508. Patnaik A. K., Liu S. K., Hurvitz A. I., McClelland A. J.: Nonhematopoietic neoplasms in cats. J. Natl. Cancer Inst. 1975, 54:855-860.

Peters S.L.: Inventor Michelson offers \$million offered for a way to sterilize pets. USA Today $10 / 15 / 2008$

Pesteanu-Somogyi L. D., Radzai C., Pressler B. M.: Prevalence of feline infectious peritonitis in specific cat breeds. J. Feline Med. Surg. 2006, 8:1-5.

Potter K., Hancock D. H., Gallina A. M.: Clinical and pathologic features of endometrial hyperplasia, pyometra, and endometritis in cats: 79 cases (1980-1985). J. Am. Vet. Med. Assoc.1991, 198:1427-1431.

Prahl A., Guptill L., Glickman N. W., Tetrick M., Glickman L. T.: Time trends and risk factors for diabetes mellitus in cats presented to veterinary teaching hospitals. J. Feline Med. Surg. 2007, 9:351-358.

Rohrbach B. W., Legendre A. M., Baldwin C. A., Lein D. H., Reed W. M., Wilson R. B.: Epidemiology of feline infectious peritonitis among cats examined at veterinary medical teaching hospitals. J. Am. Vet. Med. Assoc. 2001, 218:1111-1115.

Root M. V., Johnston S. D., Johnston G. R., Olson P. N.: The effect of prepuberal and postpuberal gonadectomy on penile extrusion and urethral diameter in the domestic cat. Vet. Radiol. Ultrasound. 1996, 37:363-366.

Root M. V., Johnston S. D., Olson P. N.: The effect of prepuberal and postpuberal gonadectomy on radial physeal closure in male and female domestic cats. Vet. Radiol. Ultrasound. 1997, 38:42-47.

Scarlett J. M., Donoghue S.: Associations between body condition and disease in cats. J. Am. Vet. Med. Assoc. 1998, 212:1725-1731.

Schmidt R., Langham R.: A survey of feline neoplasms. J. Am. Vet. Med. Assoc.1967, 151:1325-1328.

Spain C. V., Scarlett J. M., Houpt K. A.: Long-term risks and benefits of early-age gonadectomy in cats. J. Am. Vet. Med. Assoc. 2004, 224:372-379.

Stubbs W. P., Bloomberg M. S., Scruggs S. L., Shille V. M., Lane T. J.: Effects of prepubertal gonadectomy on physical and behavioral development in cats. J. Am. Vet. Med. Assoc.1996, 209:1864-1871. 
Wright J. C., Amoss R. T.: Prevalence of house soiling and aggression in kittens during the first year after adoption from a humane society. J. Am. Vet. Med. Assoc. 2004, 224:17901795.

Korrespondenz

PD Dr. I.M. Reichler

Abteilung für Kleintierfortpflanzung, Klinik für Fortpflanzungsmedizin, Vetsuisse-Fakultät Universität Zürich

Winterthurerstr.260, CH-8057 Zürich

Fax 00414463589 40, email: ireichler@ vetclinics.uzh.ch 
Dateiname: KätzinnenKaterKastration20090921.doc

Verzeichnis: $\quad$ C:IDokumente und Einstellungenliris\Eigene

Dateien\Veröffentlichungen\Eigene\SAT

Vorlage:

C:IDokumente und

Einstellungen $\backslash$ iris $\backslash$ Anwendungsdaten $\backslash$ Microsoft $\backslash$ Vorlagen $\backslash$ Normal.dot Titel:

Vor- und Nachteile der Kastration von Kätzinnen und Katern

Thema:

Autor:

Stichwörter:

Kommentar:

Erstelldatum:

21.09.2009 14:33:00

Änderung Nummer: 2

Letztes Speicherdatum: $\quad 21.09 .2009$ 14:33:00

Zuletzt gespeichert von: iris

Letztes Druckdatum: 27.01.2011 11:23:00

Nach letztem vollständigen Druck

Anzahl Seiten: 11

Anzahl Wörter: 14.983 (ca.)

Anzahl Zeichen: 94.400 (ca.) 